\title{
Amperometric Morphine Detection Using Pt-Co Alloy Nanowire Array-modified Electrode
}

\author{
Manlan Tao, Feng Xu, Yueting Li, Quanqing Xu, Yanbing Chang, Zaisheng Wu, ${ }^{\dagger}$ and Yunhui Yang* \\ College of Chemistry and Chemical Engineering, Yunnan Normal University, Kunming 650092, P. R. China \\ *E-mail:yyhui2002@yahoo.com.cn \\ †State Key Laboratory of Chemo/Biosensing and Chemometrics, Hunan University, Changsha 410082, P. R. China \\ Received November 25, 2009, Accepted May 19, 2010
}

\begin{abstract}
Pt-Co alloy nanowire array was directly synthesized by electrochemical deposition with polycarbonate template at $-1.0 \mathrm{~V}$ and subsequent chemical etching of the template. The use of Pt-Co alloy nanowire array-modified electrode (PtCo NAE) for the determination of morphine (MO) is described. The morphology of the Pt-Co alloy nanowire array has been investigated by scanning electron microscopy (SEM) and energy disperse X-ray spectroscopy (EDS) analysis), respectively. The resulting Pt-Co NAE offered a linear amperometric response for morphine ranging from $2.35 \times 10^{-5}$ to $2.39 \times 10^{-3} \mathrm{M}$ with a detection limit of $7.83 \times 10^{-6} \mathrm{M}$ at optimum conditions. This sensor displayed high sensitivity and long-term stability.
\end{abstract}

Key Words: Morphine, Pt-Co alloy, Nanowire array, Amperometric, Electrode

\section{Introduction}

Morphine, a well-known potent narcotic analgesic and the active metabolite derived from heroin (3,6-diacetylmorphine), has been reported to influence various immune functions and cause disruption in the central nervous system. It is frequently used to relieve severe pain in patients, especially those undergoing a surgical procedure. However, morphine presents toxic symptoms when used to excess or abused. Morphine is extensively metabolized to its morphine-3-glucuronide (M3G), with normorphine (NM) and morphine-6-glucuronide (M6G) as minor metabolites, irrespective of the species or administration route. ${ }^{1,2}$ Various analytical methods have been developed for the determination of morphine including gas chromatography/ mass spectrometry, ${ }^{3}$ liquid chromatography ${ }^{4}$ and liquid chromatography-mass spectrometry. ${ }^{5,6}$ However, those methods are time-consuming and very costly. Compared to other options, electro-analysis has the advantages of simplicity and high sensitivity, but the use of bare electrodes for electrochemical measurements has a number of limitations, such as slow electron transfer reaction and electrode fouling problems. Jin et al. have prepared a cobalt hexacyanoferrate $(\mathrm{CoHCF})$ chemically modified electrode (CME) for amperometric determination of morphine. ${ }^{7}$ Ho et al. have used a Prussian blue modified- indium tin oxide electrode to determine $\mathrm{MO} .{ }^{8}$ Yeh and Ho have reported an amperometric MO sensor based on molecularly imprinted electrode. ${ }^{9}$ Pournaghi-Azar and Saadatirad have reported the use of prussian blue film modified-palladized aluminum electrode (PB/Pd-Al) prepared by a simple and rapid electroless method for the determination of codeine (CO) and morphine (MO) simultaneously. ${ }^{10}$ Fast, sensitive, simple, inexpensive, and specific method for the quantitative determination of MO is still highly required.

Recently, nanomaterials have become the focus of scientific researchers because of their unique electronic, optical, and catalytic properties. ${ }^{11-14}$ Especially, metallic alloys nanowires have been of considerable interest in the field of catalysis and sensors because they often exhibit better catalytic properties than monometallic counterparts. ${ }^{15,16}$ Sun et al. ${ }^{16}$ have reported a Pt-Pb nanowire array electrode for enzyme-free glucose detection. S. Franz et al. ${ }^{17}$ have made Pt-Co alloys with microelectrodeposition for micromagnetic applications.

In this paper, the Pt-Co alloy nanowire array was synthesized in polycarbonate (PC) template by means of direct electrodeposition technique at $-1.0 \mathrm{~V}$. Furthermore, considering its good catalysis to MO, the Pt-Co alloy nanowire array-modified electrode (Pt-Co NAE) was used for the determination of MO with chronoamperometry. The major advantages of Pt-Co NAE are the simplicity, rapidity and miniaturization in preparation, high stability of the Pt-Co alloy surface and its effective catalytic activity for the electro-oxidation of MO.

\section{Experimental}

Chemicals. Nuclepore track-etch polycarbonate (PC) templates $(0.2 \mu \mathrm{m})$ were provided by Whatman (Anodisc $25 \mathrm{~mm}$ ). $\mathrm{Co}\left(\mathrm{NH}_{2} \mathrm{SO}_{3}\right)_{2}$ was supplied by Jiangxi Domestic capital chemical Co., Ltd (Jiangxi, China). $\mathrm{Pt}\left(\mathrm{NH}_{3}\right)_{2}\left(\mathrm{NO}_{2}\right)_{2}$ was purchased from Shanghai Jiuyue chemical Co., Ltd (Shanghai, China). Morphine hydrochloride (MO hydrochloride) was from the material evidence identification center of Ministry of Public Security (Beijing, China). A 0.2 M phosphate buffer (PB) solution was prepared using $\mathrm{Na}_{2} \mathrm{HPO}_{4}$ and $\mathrm{NaH}_{2} \mathrm{PO}_{4}$ and adjusted to $\mathrm{pH}$ 6.5. All of the other chemicals were of analytical grade and used as received. All solutions were prepared with doubly distilled water.

Instrumentation. Cyclic voltammetric and chronoamperometry measurements were carried out on XJP-821(C) polarograph (Jiangsu, China). Scanning electron microscopy (SEM) was performed on XL30ESEM-TMP microscope (Philips, Holand). Energy dispersive X-ray spectroscopy (EDS) was performed using an energy dispersive analysis system of X-ray 
(EDAX, USA). A three-electrode cell $(10 \mathrm{~mL})$ with the Pt-Co alloy nanowire array-modified electrode as the working electrode, a saturated calomel electrode (SCE) as reference electrode and platinum foil electrode as counter electrode was used. All potentials were measured and reported versus the SCE. The measurements were carried out at $25 \pm 1{ }^{\circ} \mathrm{C}$.

Synthesis of Pt-Co alloy nanowire. To make the template conductive, a thin film of $\mathrm{Au}(\sim 30 \mathrm{~nm})$ was first sputtered onto one surface of the PC membrane using the pumped vacuum spray gold device of SEM. The PC template sputtered with thin gold film was connected with a copper wire $(5 \mathrm{~cm})$ using graphite conductive adhesive. The preparation of PC template was completed after the conductive adhesive dried at room temperature.

Before the deposition, the PC template was first placed in the electroplating solution for $10 \mathrm{~min}$ in order to allow the solution to penetrate throughout the template. According to the report of S. Franz et al., ${ }^{17}$ electrodeposition was carried out in a complex solution containing $0.1 \mathrm{M}$ cobalt sulphamate, $0.01 \mathrm{M}$ $\mathrm{Pt}\left(\mathrm{NH}_{3}\right)_{2}\left(\mathrm{NO}_{2}\right)_{2}, 0.1 \mathrm{M}$ ammonium citrate, and $0.1 \mathrm{M}$ glycine by applying certain potential of $-1.0 \mathrm{~V}$. The electrolyte $\mathrm{pH}$ was maintained at 8 by addition of either sodium hydroxide or sulphamic acid if necessary. After deposition of $30 \mathrm{~min}$, the synthesis of Pt-Co alloy nanowire was completed. In contrast, Pt nanowires were prepared in $0.1 \mathrm{M} \mathrm{KCl}$ solution containing 2 $\mathrm{mM} \mathrm{H}_{2} \mathrm{PtCl}_{6}$ at $-1.0 \mathrm{~V}$.

Fabrication of Pt-Co alloy nanowire array-modified electrode. Glassy carbon electrode (GCE) (3 mm diameter) was used as the substrate electrode. Before use, it was first polished with $1.0,0.3$ and $0.05 \mu \mathrm{m}$ alumina slurry and rinsed thoroughly with absolute alcohol and distilled water in ultrasonic bath, and dried in air at room temperature.

A piece of the deposited PC template was placed gold-side down onto the surface of the GCE. Then, chloroform was dropped onto PC membrane for several times to dissolve PC membrane and release Pt-Co alloy nanowire. After the volatilizing of the chloroform, the film of Pt-Co alloy nanowire were kept onto the surface of the GCE with good stability and conductivity. The electrode was washed successively with ethanol and water. For comparison, Pt nanowires array-modified electrode was prepared using same process. Conventional bare Pt electrode was also used as comparison after physically polishing with alumina slurry of different size.

Electrochemical measurements. The cyclic voltammetric behaviors of the modified electrode in $0.2 \mathrm{M}$ PB solution ( $\mathrm{pH}$ 6.5 ) before and after adding morphine were investigated at room temperature from $0 \mathrm{~V}$ to $+1.2 \mathrm{~V}$ (versus $\mathrm{SCE}$ ). Amperometric measurements were carried out at $0.7 \mathrm{~V}$ in a stirred solution with a steady-state background current first recorded before stock morphine hydrochloride standard solution was added into the buffer solution.

\section{Results and Discussion}

Characteristics of Pt-Co alloy nanowire array. The morphology of the nanowires array was characterized by SEM (Fig. 1A).The average diameter of nanowire is about $250 \mathrm{~nm}$, which corresponds to the size of the nanopore in the template. The average length of the nanowires is about $5 \mu \mathrm{m}$. It can also be seen
(A)

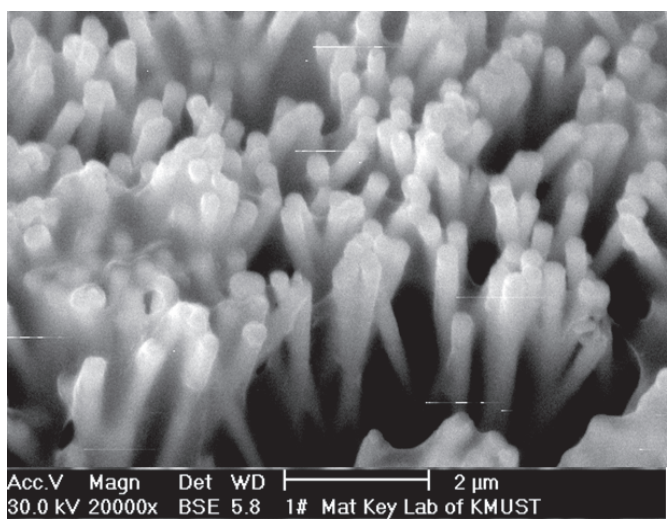

(B)

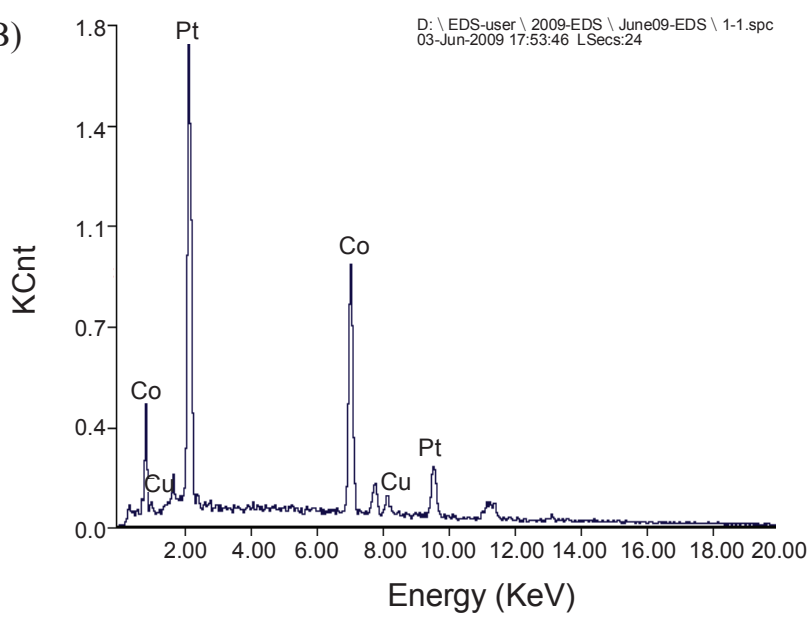

Figure 1. SEM image of the Pt -Co alloy nanowire array after PC template was dissolved for $15 \mathrm{~min}$ (A) and EDS of the Pt -Co alloy nanowire array (B).

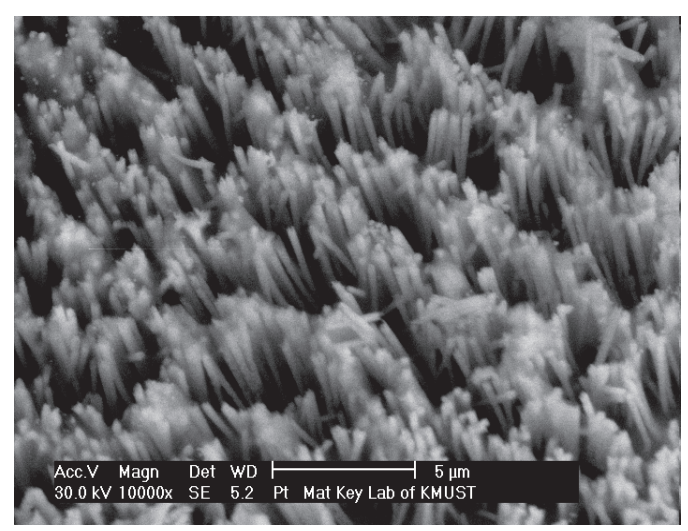

Figure 2. SEM images of the platinum nanowire array after PC template was dissolved for $15 \mathrm{~min}$.

from the image that each nanowire is vertically, making each nanowire work as an individual nanoelectrode. EDS analysis also confirmed the formation of Pt-Co alloys (Fig. 1B). The morphology of the platinum nanowire array was also characterized by SEM (Fig. 2). The average diameter of the nanowires was about $250 \mathrm{~nm}$ with the length of about $2.5 \sim 3 \mu \mathrm{m}$.

Fig. 3 shows the SEM images of the Pt-Co alloy nanowire array with different dissolved time by chloroform. As it can be 

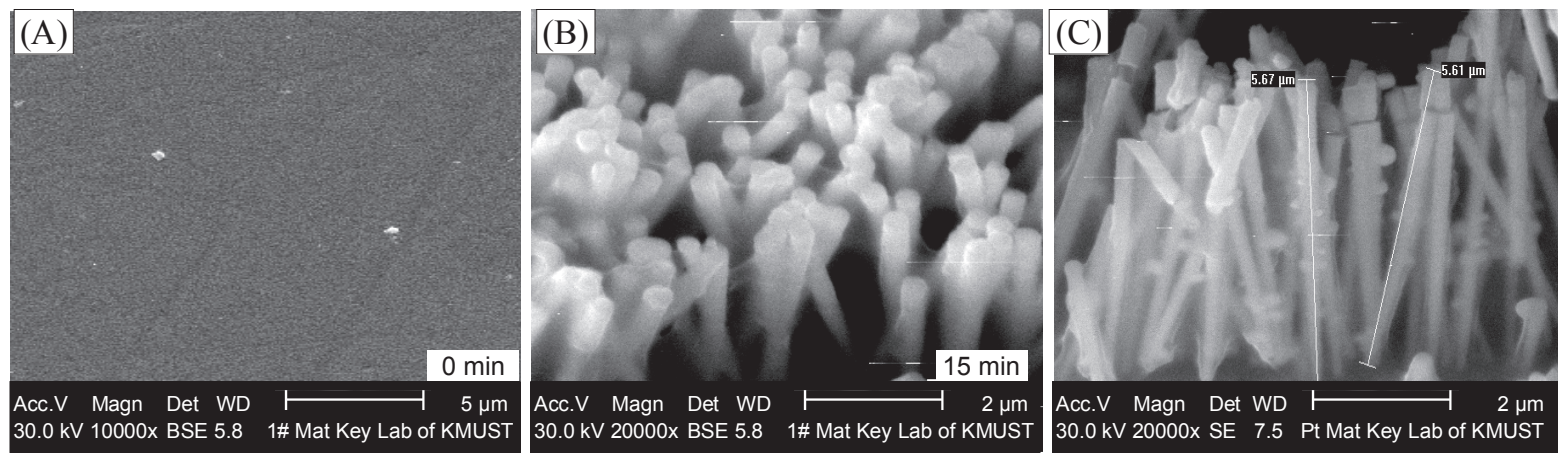

Figure 3. SEM images of the Pt -Co alloy nanowire array on PC template with different dissolving time. A: 0 min B: 15 min C: 30 min.

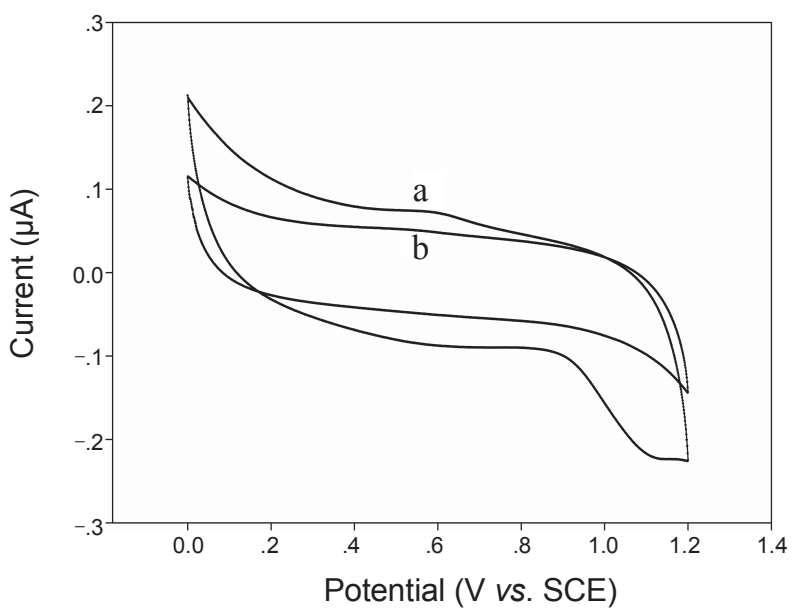

Figure 4. Cyclic voltammograms of the Pt-Co NAE in buffer solution before (b) and after (a) adding $0.26 \mathrm{mM}$ morphine (scan rate: $100 \mathrm{mV}$ $\left.\mathrm{s}^{-1}\right)$.

seen, when PC template was not dissolved, Pt -Co alloy nanowire array can not be released (Fig. 3A). When dissolved time was $15 \mathrm{~min}$ (Fig. 3B), the Pt -Co nanowire stands vertically on the substrate surface, and the diameter is rather uniformly distributed around $(230 \pm 20 \mathrm{~nm})$. As expected, these nanowires assume the shape and dimensions of the pores. Furthermore, there are apparently no macroscopic defects in the nanowire. However, when dissolved time was too long (30 min), PC membrane was dissolved so completely that Pt -Co nanowire released from PC membrane were not vertically oriented, and the nanowire array was destroyed (Fig. 3C). Therefore, the dissolved time of 15 min was adopted in the subsequent work.

Electrochemical properties of Pt-Co NAE. Voltammetric experiments were used to evaluate the electrochemistry of the Pt-Co NAE. Fig. 4 shows the cyclic voltammograms (CV) obtained with the Pt-Co NAE in unstirring $0.2 \mathrm{MPB}(\mathrm{pH} 6.5)$ solution without (b) and with (a) $0.26 \mathrm{mM}$ morphine. Only background current could be observed in Fig. 4 (b). In the presence of $0.26 \mathrm{mM}$ morphine (Fig. 4 (a)), the anodic peak current increased at $0.543 \mathrm{~V}$, indicating that Pt-Co NAE exhibits electrocatalytic activity toward morphine. Due to the higher background, the CV curves of Pt-Co NAE seem to have weak redox peaks compared with the $\mathrm{CV}$ curves of Prussian blue-modified indium tin oxide electrode ${ }^{8}$ and molecularly imprinted polymer-

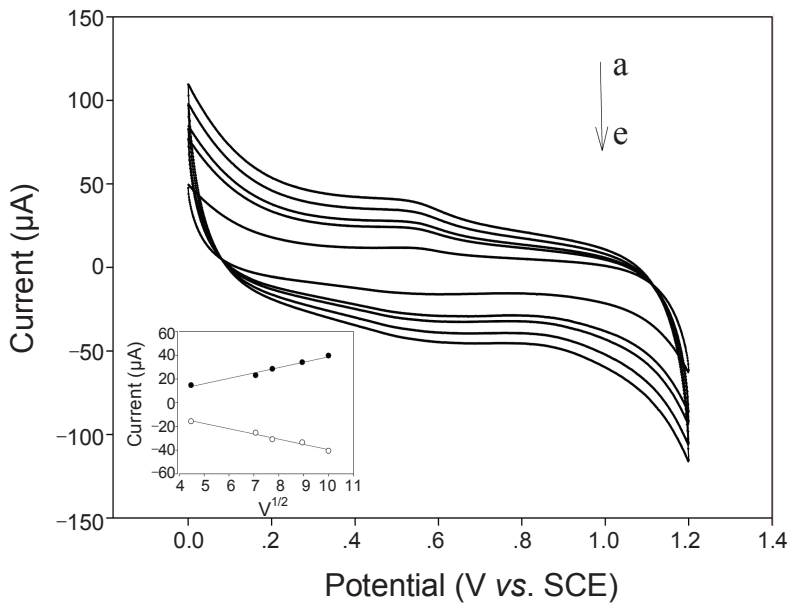

Figure 5. Cyclic voltammograms of the Pt-Co NAE at different scan rate of a: 100, b: 80, c: 50, d: 40 and e: $20 \mathrm{mV} \mathrm{s}^{-1}$ Inset: plots of oxidation peak current versus $\mathrm{v}^{1 / 2}$.

modified electrode. ${ }^{9}$

Fig. 5 shows CV of the Pt-Co NAE in 0.2M PB (pH 6.50) containing $0.26 \mathrm{mM}$ morphine at different scan rates. A linear relationship between the redox peak currents and the square root of scan rate $\left(\mathrm{v}^{1 / 2}\right)$ (see the inset of Fig. 5) indicates that the peak current is diffusion-controlled.

To discern the role of individual components and possible synergy between $\mathrm{Pt}$ and $\mathrm{Co}$, four electrodes were investigated by chronoamperometry with successive addition of the same amounts $(0.26 \mathrm{mM})$ of morphine in $\mathrm{PB}$ (pH 6.5) (Fig. 6a: bare GCE; b: conventional Pt electrode; c: Pt NWEs; d: Pt-Co NAE). Noticeably, the sensitivity of Pt-Co NAE was the largest among that of four electrodes, indicating that the Pt- Co alloy can enhance the response. The results prove that Pt- Co alloy exhibit better catalytic properties than monometallic counterparts. ${ }^{16}$

Optimization of amperometric detection conditions. The effect of applied potential on the response of Pt-Co NAE was investigated in the presence of $0.26 \mathrm{mM}$ morphine and was shown in Fig. 7 With the increasing of potential from $0.46 \mathrm{~V}$ to $0.88 \mathrm{~V}$, the sensitivity of the electrode increased significantly and reached a plat at 0.68 to $0.81 \mathrm{~V}$. The inset of Fig. 7 showed background current in $0.2 \mathrm{M} \mathrm{PB}$ at each potential. To obtain high signal-tonoise $(\mathrm{S} / \mathrm{N})$ ratio, a potential of $0.70 \mathrm{~V}$ ( $v s . \mathrm{SCE})$ was selected as the applied potential for amperometric measurements. 


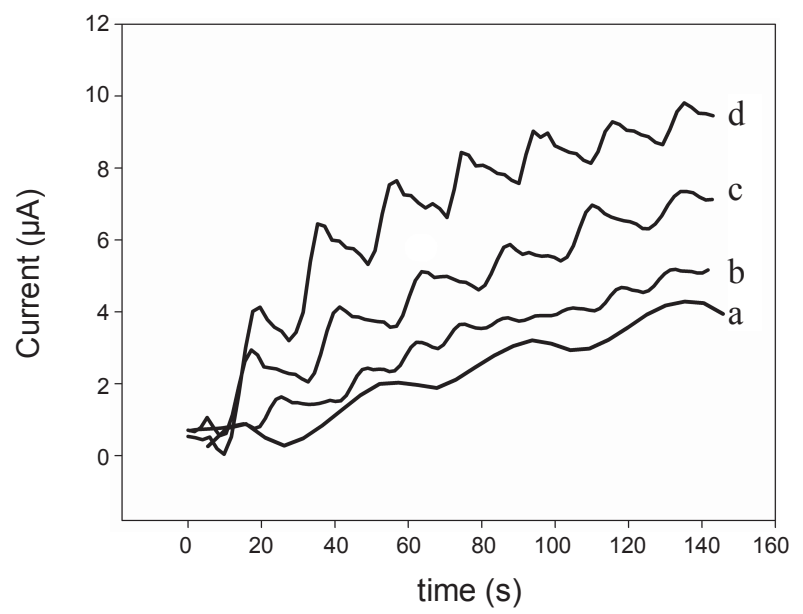

Figure 6. Dynamic responses of different electrodes ((a) bare GCE; (b) bare Pt; (c) Pt NAE; (d) Pt-Co NAE) for successive addition of the same mount $(0.26 \mathrm{mM})$ morphine in $0.2 \mathrm{M}$ phosphate buffer solution $(\mathrm{pH}$ $6.50)$.

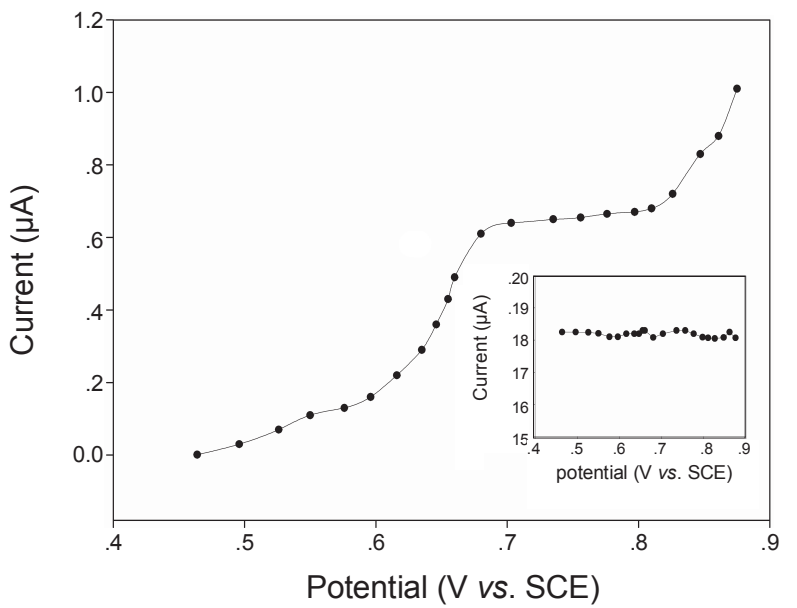

Figure 7. Effect of applied potential on the response of the Pt-Co NAE in $0.2 \mathrm{M}$ phosphate buffer solution ( $\mathrm{pH} 6.50$ ) containing $0.26 \mathrm{mM}$ morphine. Inset: plots of background current versus potential.

The influence of the $\mathrm{pH}$ of the assay solution over the range 5.92 - 8.64 on the chronoamperometry response of the Pt-Co NAE to morphine at a fixed concentration of $0.2 \mathrm{M}$ in PB solution was also investigated. Fig. 8 showed that the optimum $\mathrm{pH}$ was 6.50. According to the report of Pournaghi-Azar. ${ }^{10}$ the electro-catalytical oxidization pathway of $\mathrm{MO}$ at neutral media can be shown as follows:

$$
\begin{aligned}
& 2 \mathrm{MO} \rightarrow 2 \mathrm{MO} \cdot+2 \mathrm{e}^{-}+2 \mathrm{H}^{+} \\
& 2 \mathrm{MO} \cdot \rightarrow \mathrm{PMO}
\end{aligned}
$$

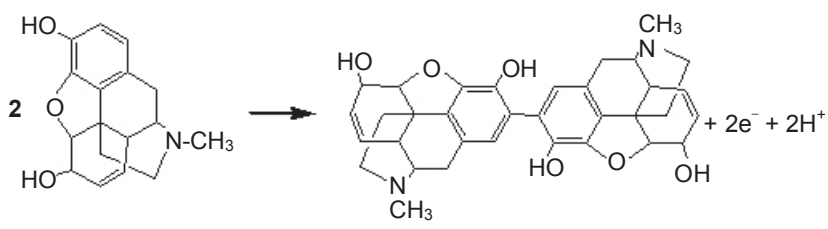

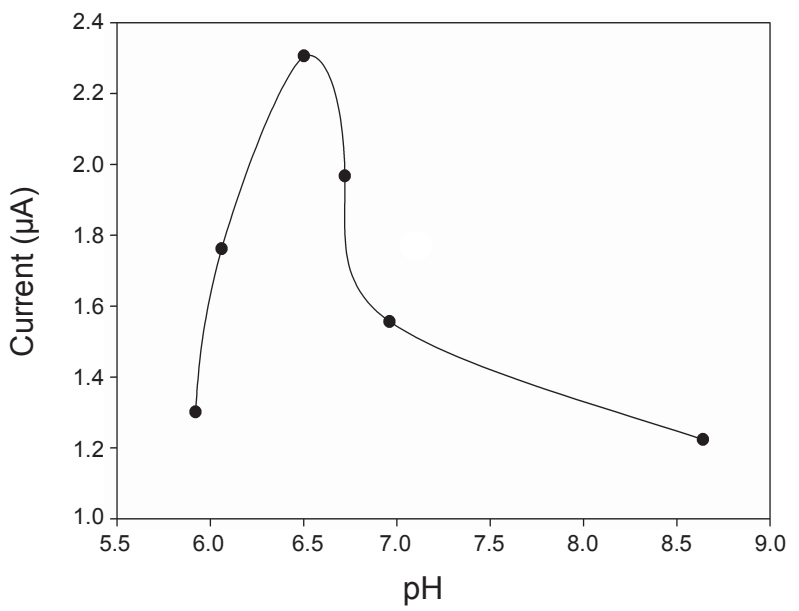

Figure 8. Effect of $\mathrm{pH}$ on the response of the Pt-Co NAE.

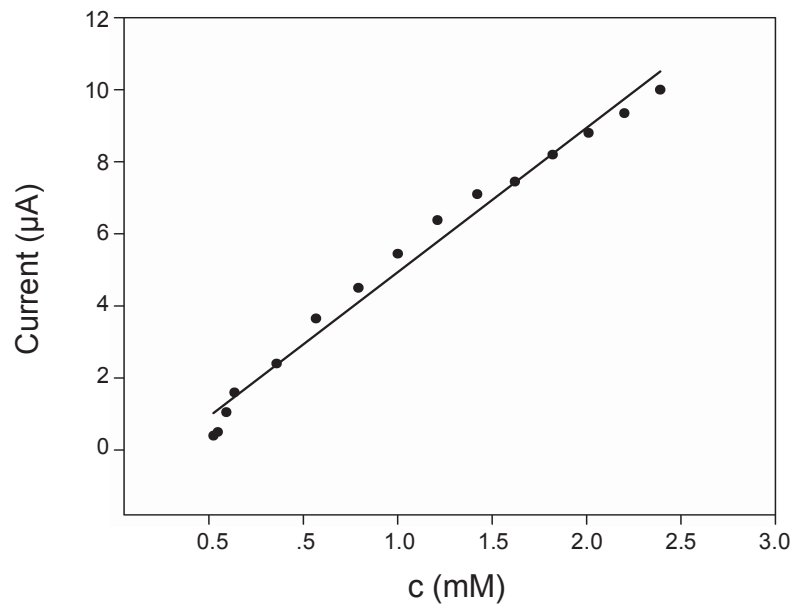

Figure 9. The calibration of Pt-Co NAE toward morphine.

Low $\mathrm{pH}$ is not beneficial to the reaction. Therefore, $\mathrm{pH} 6.50$ was fixed for the rest of the experiments.

Morphine detection performance. The Pt-Co NAE exhibited a rapid and sensitive response to the change of morphine concentration. This indicated the nice electrocatalytic behavior of the Pt-Co NAE. The calibration curve for morphine was shown in Fig. 9. The linear range is from $2.35 \times 10^{-5}$ to $2.39 \times 10^{-3} \mathrm{M}$ with a correlation coefficient of 0.987 , the regression equation was $\mathrm{I}(\mu \mathrm{A})=0.922+4.02 \mathrm{C}(\mathrm{mM})$, where I was response current, and $\mathrm{C}$ was morphine concentration. The detection limit was $7.83 \times 10^{-6} \mathrm{M}$ at a signal-to-noise $(\mathrm{S} / \mathrm{N})$ ratio 3 . The linear range is wider than the report of Ho et al. ${ }^{8}$ and Pournaghi-Azar et al. ${ }^{10}$ The detection limit is lower than Yeh and Ho's report using a molecularly imprinted polymer-modified electrode. ${ }^{9}$ The relative low detection limit was due to the Pt-Co NAE. It has been reported that the use of microelectrode arrays instead of conventional ones could improve the ratio of signal-to-noise $(\mathrm{S} / \mathrm{N})$ ratio and decrease the detection limit. ${ }^{18}$ 
Table 1. Possible interferences tested with the Pt-Co NAE

\begin{tabular}{lcc}
\hline \multicolumn{1}{c}{ Interferents } & $\mathrm{C}_{\text {interferences }} / \mathrm{C}_{\text {morphine }}$ & $\mathrm{i}_{\text {interferences }} / \mathrm{i}_{\text {morphine }}$ \\
\hline ascorbic acid & 1.88 & 3.157 \\
glucose & 9.49 & 0.706 \\
cocaine & 1.88 & 0.050 \\
uric acid & 7.97 & 0.220 \\
\hline
\end{tabular}

Table 2. Determination of morphine recovery

\begin{tabular}{ccc}
\hline $\begin{array}{c}\text { added concentration } \\
(\mathrm{mM})\end{array}$ & $\begin{array}{c}\text { found concentration } \\
(\mathrm{mM})\end{array}$ & recovery $(\%)$ \\
\hline 0.201 & 0.217 & 108.0 \\
0.257 & 0.268 & 104.3 \\
0.532 & 0.529 & 99.6 \\
\hline
\end{tabular}

The selectivity of the sensor was also assessed by determination of four possible interferences infection for the electrode. Table 1 showed that only ascorbic acid interferes with the determination of morphine because it also can be oxidized at this applied potential.

The repeatability of the response current of the Pt-Co NAE was investigated at a morphine concentration of $0.26 \mathrm{mM}$. The variation coefficient was $3.5 \%$ for five successive assays. The stability and lifetime of the electrode were investigated by measuring the electrode response with $0.26 \mathrm{mM}$ of morphine every six days. The response current of the Pt-Co NAE decreased to $87.1 \%$ after 100 days. It suggests that the stability of the electrode was good. When not in use, the electrode was stored dry at room temperature.

A preliminary evaluation of the validity of the proposed Pt-Co NAE was performed in the real serum samples. Different amount morphine were added into serum samples and determined with proposed Pt-Co NAE. The results were shown in Table 2. The recoveries were satisfactory. This result showed the proposed method may provide a feasible alternative tool for determining morphine in human serum.

\section{Conclusions}

In this paper, Pt-Co alloy nanowire array was synthesized by electrodeposition with polycarbonate template and subsequent chemical etching of the template. Based on the electrochemistry oxidation of morphine at $0.7 \mathrm{~V}$, the Pt-Co alloy nanowire array- modified electrode was used to determine morphine. The Pt-Co alloy nanowire array improves analytical performances of the corresponding electrode compared to Pt nanowire array-modified electrode and the conventional Pt electrodes, specifically. The other possible interferents such as cocaine, glucose have no significant interference. The determination of morphine in real serum samples was satisfactory. The Pt-Co NAE displayed high sensitivity, quick response to morphine, good stability, wide linear range, better detection limit. The simple and fast fabrication of the electrode makes it superior to other techniques.

Acknowledgments. This work was supported by the National Natural Science Foundation of China (Grants No. 20865006), the Foundation of Science Commission of Yunnan Province (No.2006B0028M) and the Foundation of Department of Education of Yunnan Province (No.07Z10087).

\section{References}

1. Milne, R. W.; Nation, R. L.; Somogyi, A. A.; Crugten J. T. B: Biomed. Appl. 1991, 565, 457.

2. Yeh, S. Y.; Gorodetzky, C. W. J. Pharm Sci. 1977, 66, 1288.

3. Pötscha, L.; Skoppb, G. Forensic Sci. Int. 1996, 81, 95.

4. Hara, S.; Mochinaga, S.; Fukuzawa, M.; Ono, N.; Kuroda, T. Anal. Chim. Acta 1999, 387, 121.

5. Sooyeun, L.; Rosa, C.; Sue, P. Forensic Sci. Int. 2009, 183, 74.

6. Schanzle, G.; Li, S.; Mikus, G.; Hofmann, U. B: Biomed. Appl. 1999, 721, 55.

7. Xu, F.; Gao, M.; Wang, L.; Zhou T.; Jin, L.; Jin, J. Talanta 2002, $58,427$.

8. Ho, K. C.; Chen, C. Y.; Hsu, H. C.; Chen, L. C.; Shiesh, S. C.; Lin, X. Z. Biosens. Bioelectron. 2004, $20,3$.

9. Yeh, W. M.; Ho, K. C. Anal. Chim. Acta 2005, 542, 76.

10. Pournaghi-Azar, M. H.; Saadatirad, A. J. Electroanal. Chem. 2008, 622, 293.

11. Yang, G. M.; Tan, L.; Shi, Y.; Wang, S. P.; Lu, X. X.; Bai, H. P.; Yang, Y. H. Bull. Korean Chem. Soc. 2009, 30, 454.

12. Xue, F. H.; Fei, G. T.; Wu, B.; Cui, P.; Zhang, L. D. J. Am. Chem. Soc. 2005, 127, 15348.

13. Wen, D.; Liu, Y.; Yang, G. C.; Dong, S. J. Electrochimica Acta 2007, 52, 5312.

14. Ma, H. X.; Yang, G. B.; Yu, L. G.; Zhang, P. Y. Surf. Coat. Technol. 2008, 202, 5799.

15. Luo, J.; Njoki, N.; Lin, Y.; Wang, L.; Zhong, C. Langmuir 2006, 22, 2892.

16. Bai, Y.; Sun, Y. Y.; Sun, C. Q. Biosens. Bioelectron. 2008, 24, 579.

17. Cavallotti, P. L.; Bestetti, M.; Franz, S. Electrochim. Acta 2003, 48, 3013.

18. Puganova, E. A.; Karyakin, A. A. Sens. Actuators, B: Chemical. 2005, 109, 167. 